\title{
Increased levels of intramuscular cytokines in patients with jaw muscle pain
}

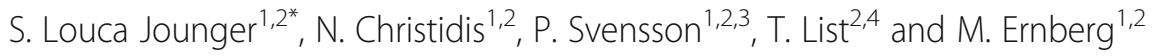

\begin{abstract}
Background: The aim of this study was to investigate cytokine levels in the masseter muscle, their response to experimental tooth-clenching and their relation to pain, fatigue and psychological distress in patients with temporomandibular disorders (TMD) myalgia.

Methods: Forty women, 20 with TMD myalgia (Diagnostic Criteria for TMD) and 20 age-matched healthy controls participated. Intramuscular microdialysis was performed to sample masseter muscle cytokines. After 140 min (baseline), a 20-minute tooth-clenching task was performed (50\% of maximal voluntary contraction force). Pain (Numeric rating scale 0-10) and fatigue (Borg's Ratings of Perceived Exertion 6-20) were assessed throughout microdialysis, while pressure-pain thresholds (PPT) were assessed before and after microdialysis. Perceived stress (PSS-10) and Trait Anxiety (STAl) were assessed before microdialysis.

Results: The levels of IL-6, IL-7, IL-8 and IL-13 were higher in patients than controls (Mann Whitney U-test; $P^{\prime} s<0$. 05) during the entire microdialysis. IL-6, IL-8 and IL-13 changed during microdialysis in both groups (Friedman; $\left.P^{\prime} s<0.05\right)$, while IL-1 $\beta, I L-7$ and GM-CSF changed only in patients $\left(P^{\prime} s<0.01\right)$. IL-6 and IL-8 increased in response to tooth-clenching in both groups (Wilcoxon test; $P^{\prime} s<0.05$ ), while IL-7, IL-13 and TNF increased only in patients ( $\left.P^{\prime} s<0.05\right)$. Patients had higher pain and fatigue than controls before and after tooth-clenching $(P<0.001)$, and lower PPTs before and after microdialysis $(P<0.05)$. There were no correlations between cytokine levels, pain or fatigue. Also, there were no differences in stress or anxiety levels between groups.

Conclusions: In conclusion, the masseter levels of IL-6, IL-7, IL-8 and IL-13 were elevated in patients with TMD myalgia and increased in response to tooth-clenching. Tooth-clenching increased jaw muscle pain and fatigue, but without correlations to cytokine levels. This implies that subclinical muscle inflammation may be involved in TMD myalgia pathophysiology, but that there is no direct cause-relation between inflammation and pain.
\end{abstract}

Keywords: Cytokines, Bruxism, Masseter muscle, Myalgia, Temporomandibular disorders (TMD)

\section{Background}

Temporomandibular disorders (TMD) are the most common chronic pain conditions in the orofacial region, affecting approximately $10-15 \%$ of the adult population [1] and twice as many women as men [2]. The most common subtype is TMD myalgia with jaw muscle pain that is increased by function, pain on palpation, pain referral, restricted mouth opening, and headache [3, 4]. The etiology of TMD and the higher prevalence among women is not well understood.

\footnotetext{
* Correspondence: sofia.louca@ki.se

${ }^{1}$ Section for Orofacial Pain and Jaw Function, Department of Dental Medicine, Karolinska Institutet, SE 14104, Huddinge, Sweden

${ }^{2}$ Scandinavian Center for Orofacial Neurosciences (SCON), Huddinge, Sweden Full list of author information is available at the end of the article
}

One hypothesis is that excessive tooth-clenching/ grinding might contribute by disturbing the local blood flow in overloaded muscles, leading to ischemia [5]. Epidemiological studies show greater odds of having TMD myalgia when self-reported tooth-clenching is present [6-8]. Ischemia releases neuroactive and inflammatory biomarkers, such as neuropeptides, bradykinin, protons, serotonin (5-HT), glutamate and cytokines that may activate and sensitize nociceptors on peripheral sensory afferents to induce muscle pain and allodynia $[9,10]$. Repeated muscle activity may then maintain chronic muscle pain by temporal summation [11]. Previous studies have shown that intense chewing induced pain and fatigue in pain-free healthy participants with similar, but 
transient symptoms as in TMD myalgia patients [12-14]. This observation may suggest that, at least a subset of M-TMD pain patients could be more alike an exerciseinduced muscle pain probably caused by ischemia and an accumulation of metabolic biomarkers in the masticatory muscles [12-14]. In another study, experimental toothclenching increased jaw muscle pain in patients with M-TMD and caused low levels of pain in controls. Patients with M-TMD had higher levels of 5-HT during the entire experiment, but did not increase in response to tooth-clenching, suggesting that other algesic substances might be released and activate nociceptors that are most likely already sensitized by 5 -HT [15].

Studies have shown that several cytokines play a role in acute inflammatory muscle pain and in some chronic muscle pain conditions. There are both pro- and antiinflammatory cytokines interacting with each other in a balanced matter in order to fight an infection and promote wound healing, and they are often released in a cascade in response to tissue damage. The proinflammatory cytokines can initiate an inflammatory response by recruiting other cytokines, macrophages, t-cells and b-cells, initiating the inflammatory response, while anti-inflammatory cytokines can reduce and promote healing by controlling the cytokine response [16].

For example, patients with various chronic pain conditions (neuropathic, nociceptive and mixed pain) had higher serum level of interleukin (IL)-6, IL-1 $\beta$, IL-2, tumor necrosis factor (TNF), and interferon gamma (IFN- $\gamma$ ) than healthy controls, which correlated with pain intensity [17], and increased cerebrospinal fluid (CSF) and plasma level of IL-8 were reported in patients with widespread pain $[18,19]$. In patients with TMD pain increased levels of IL-1 $\beta$, IL-6, IL-10, TNF, IL-1ra, and monocyte attractant protein-1 (MCP-1) have been reported $[19,20]$.

Also, muscle levels of TNF and IL-1 $\beta$ were increased in trapezius trigger points in patients with myofascial pain $[21,22]$, and IL-6 increased in the painful trapezius muscle of patients with whiplash-associated disorders (WAD) [23]. Muscle and plasma levels of IL-6 and IL-8 increase in response to exercise $[24,25]$. However, serum IL-10 showed a blunted response to exercise in fibromyalgia [26].

Regardless of their potential role in the development and maintenance of some chronic pain conditions, little is known about the peripheral involvement of cytokines in TMD myalgia. The aim of this study was therefore to compare the levels of the pro- and anti-inflammatory cytokines IL-1 $\beta$, IL-2, IL-4, IL-5, IL-6, IL-7, IL-8, IL-10, IL-12, IL-13, TNF, IFN- $\gamma$, and GM-CSF in the masseter muscle between TMD myalgia patients and healthy controls and the effects of a repetitive tooth-clenching task on their release and relation to masseter pain intensity, fatigue, and pressure-pain thresholds (PPT). Furthermore, perceived stress and anxiety may also be involved in the pathophysiology of TMD pain [27]. Therefore, questionnaires were used to estimate the potential correlation between biomarkers and psychological variables.

We hypothesized that muscle level of the cytokines would be higher in patients than controls, increase in response to a repetitive tooth-clenching task in a similar manner in both groups, and correlate to pain intensity, fatigue and level of stress and anxiety.

\section{Methods \\ Participants}

Twenty women with TMD myalgia and twenty agematched healthy women were included. Participants were recruited by advertisements and among colleagues and students at the Department of Dental Medicine at the Karolinska Institutet, Huddinge, Sweden where the study was performed. Inclusion criteria for the patients were age over 18, a diagnosis of myalgia according to the Diagnostic Criteria for TMD (DC/TMD) [1] and pain lasting for over 3 months. Inclusion criteria for the controls were age over 18, good general health and no history of/or current pain from the orofacial region. Exclusion criteria for both groups were systemic muscular or joint diseases, WAD, neuropathic pain or neurological disorders, pain of dental origin, and use of analgesics of non-steroidal anti-inflammatory drugs during $48 \mathrm{~h}$ before microdialysis.

Based on a previous study $[15,28]$ the power calculation showed that inclusion of 20 participants in each group would be sufficient to detect a significant difference of $20 \%$ (SD 30\%) in biomarker levels with $80 \%$ power and a significance level of $5 \%$.

The study followed the guidelines of the Declaration of Helsinki and was approved by the Regional Ethical Review Board in Stockholm (2009/2047-32), Sweden. All participants were given written and verbal information before participating and gave their written consent. The participants were compensated upon completion of their participation.

\section{Experimental protocol}

The study used a case-control design with one session lasting for $220 \mathrm{~min}$. After inclusion, psychological distress was assessed; thereafter the maximal voluntary clenching force (MVCF), and PPT were recorded. Participants were reclined in a conventional dental chair and instructed to lie as still as possible during the experiment and to avoid talking. After baseline registrations and local anesthesia, microdialysis was performed in one of the masseter muscles. After 120-minutes (trauma phase), baseline assessments of pain intensity and fatigue were made (120-140 $\mathrm{min})$, followed by a 20-minute 
clenching task (exercise). Pain intensity and fatigue were assessed after tooth-clenching. After an additional hour (recovery) the microdialysis catheter was removed and the PPTs were again assessed (Fig. 1).

\section{Experimental tooth-clenching}

MVCF (kg) was assessed with a bite-force transducer (Aalborg University, Denmark) placed between the molars on the most suitable side, from a dental point of view. The same side was used for the clenching task and microdialysis. Participants were instructed to bite as hard as possible on the bite-force transducer for 2-3 s. The mean of three MVCF registrations was calculated. During the experimental tooth-clenching, participants were instructed to repeatedly bite $50 \%$ of their mean MVCF for 30-seconds followed by 30-seconds of rest during 20-minutes [15]. Visual feedback was used to maintain the clenching level.

\section{Assessments of pain intensity, fatigue and pressure-pain threshold}

A 0-10 numerical rating scale (NRS) was used to assess pain intensity in the masseter muscle every $20^{\text {th }}$ minute during microdialysis. The Borg Rating of Perceived Exertion Scale (6-20; RPE) was used to measure fatigue [29].

PPT was assessed with an electronic pressure algometer (Somedic Sales AB, Höör, Sweden) with a standardized pressure rate of $50 \mathrm{kPa} / \mathrm{s}$. The tip of the device was $1 \mathrm{~cm}^{2}$, covered with a $1-\mathrm{mm}$ thick rubber-pad. PPT was recorded by applying pressure on the most prominent point of the masseter muscle during contraction, and on the right index finger, which was used as an extra-cranial reference point. Participants pressed a signal-button when the sensation of pressure turned into pain [30]. The mean of three registrations was used in statistical analyzes.

\section{Psychological distress}

The Swedish version of the State-Trait Anxiety Inventory (STAI) was used to assess trait-anxiety. It contains twenty questions measuring the levels of anxiety as a personal characteristic. Scores ranges from 20 to 80 , where high scores $(>30)$ indicate higher levels of anxiety [31, 32].

Stress levels were measured with the Swedish version of the Perceived Stress Scale-14 (PSS-14) consisting of 14 stress-related questions of a general nature, including feelings and thoughts during the last month, situations in life that are perceived as stressful, and the current level of experienced stress. The total scoring is 56, and scores below 23.67 are considered normal in healthy participants [33, 34].

\section{Microdialysis}

The most prominent point of the masseter muscle, verified by palpation during contraction, was chosen for microdialysis. The skin overlying the muscle was anaesthetized with a local injection $(0.5 \mathrm{ml})$ of Lidocaine (Xylocaine $20 \mathrm{mg} / \mathrm{ml}$ ), carefully avoiding anaesthetizing the underlying muscle.

A sterile split able introducer (CMA Microdialysis AB, Solna, Sweden) was inserted intramuscularly in parallel to the muscle fibers at a 45-degree angle to a depth of approximately $40 \mathrm{~mm}$ from the skin surface $[15,35]$.

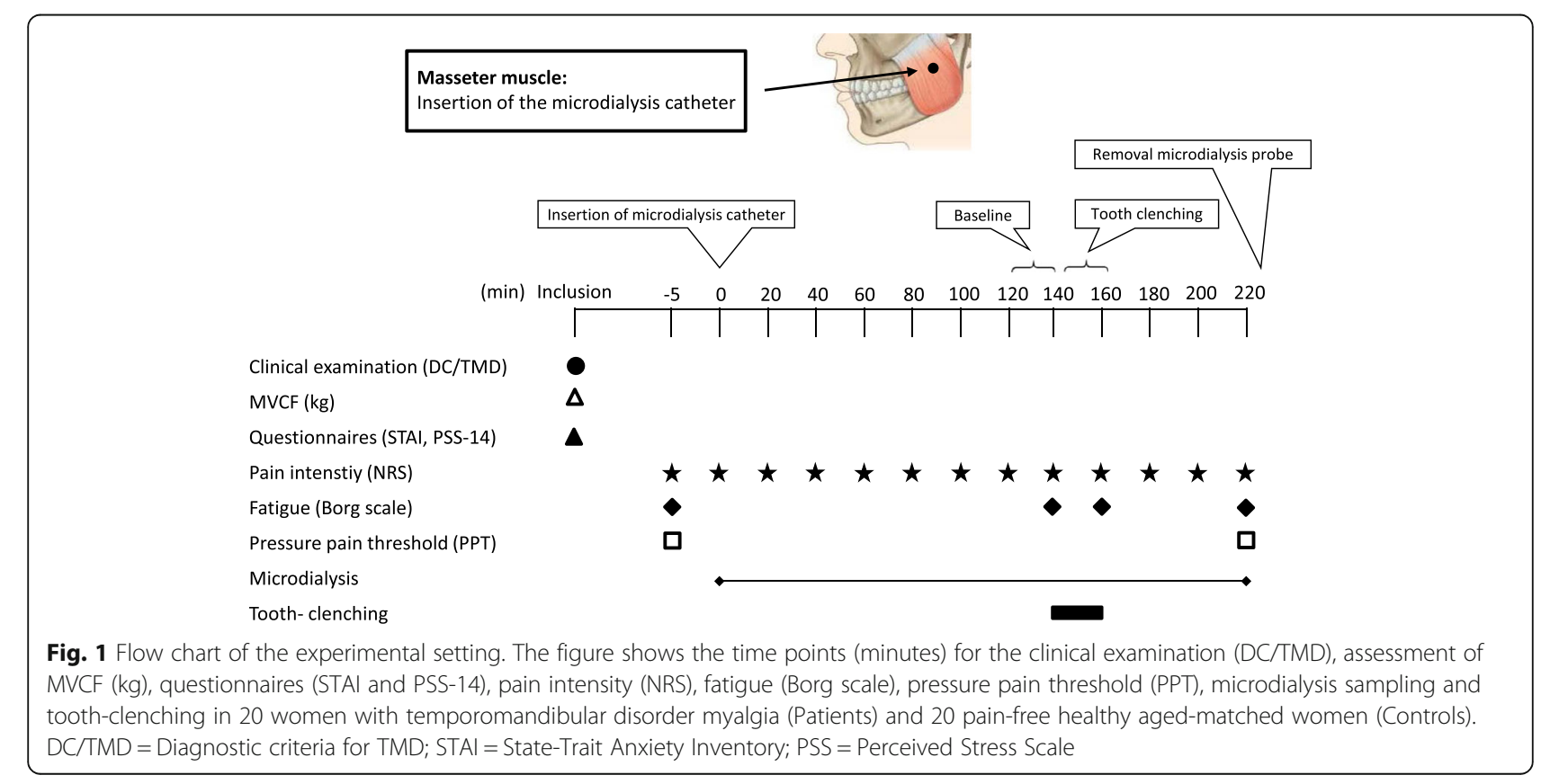


After approximately $20 \mathrm{~mm}$, a slight resistance occurred, typically felt upon penetration of the muscle fascia, and the introducer was additionally inserted $20 \mathrm{~mm}$. A sterile $100 \mathrm{kDa} 60 \mathrm{~mm}$ long microdialysis catheter with $20 \mathrm{~mm}$ membrane length (CMA71 Microdialysis AB, Solna, Sweden) was inserted to the full length, thereafter the introducer was removed by splitting the plastic tube. The catheter was perfused ( $5 \mu \mathrm{L} / \mathrm{min})$ with a RingerAcetate solution (Pharmacia \& Upjohn, Copenhagen, Denmark) containing $3 \mathrm{mmol}$ glucose and $0.5 \mathrm{mmol}$ lactate from a 2-ml syringe connected to a micro-infusion pump (CMA107, Microdialysis AB, Solna, Sweden). Samples $(100 \mu \mathrm{L})$ were collected every 20 -minutes in capped microvials and immediately frozen $\left(-80{ }^{\circ} \mathrm{C}\right)$. After microdialysis, the catheter was removed and the membrane was checked to ensure that no damaged had occurred.

\section{Analyzes of dialysate}

The cytokines were analyzed with Luminex technology using multiplex immunoassay panels (Milliplex map kit, Human High sensitivity, T-cell magnetic bead panel, 96well plate assay, Merck Millipore Darmstadt, Germany) in accordance to the manufacturer's manual.

The limit of detection (LOD) for each cytokine respectively were for IL-1 $\beta$, IL-2, IL-5, and IL-12: $0.49 \mathrm{pg}$./ mL; IL-4: 1.83 pg./mL; IL-6: 0.18 pg./mL; IL-7: 0.37 pg./ $\mathrm{mL}$; IL-8: 0.31 pg./mL, IL-10: 1.46 pg./mL; IL-13:

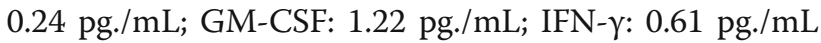
and for TNF: $0.43 \mathrm{pg} . / \mathrm{mL}$.

For each cytokine the number of samples with levels below LOD were calculated. As a quality control, cytokines with $>50 \%$ of samples below LOD were excluded from further analysis. This was based on findings from our group that some cytokines are undetectable in many individuals, making the results difficult to interpret (Ernberg et al, personal communication). Others have used similar approaches, although with less strict criteria [36].

\section{Statistics}

Data were analyzed with SigmaPlot for Windows, version 11 (Systat Software Inc. Chicago, IL, USA) and STATISTICA, StatSoft Dell Software version 12.0 (Round Rock, Texas USA). For descriptive statistics mean and standard deviation (SD) or median and interquartile range (IQR) were used. Non-parametric statistics were used since most cytokines were not normally distributed and attempts to transform data did not change this. To analyze differences in cytokine levels between groups, the average of all 11 dialysate samples (0-220 min) was calculated and compared with MannWhitney $U$-test. Friedman-test was used to analyze changes in cytokine levels over time. Wilcoxon-test compared the time points $140 \mathrm{~min}$ (BL) and 160220 min within groups. Cytokine, pain and fatigue levels after clenching (160 min) were compared between groups with Mann-Whitney $U$-test. Spearman correlations-test with Bonferroni correction for multiple testing was used to analyze correlations between cytokine levels, pain, fatigue and psychological distress. Differences in STAI-trait and PSS-14 scores between groups and differences in PPTs between sides before and after microdialysis were analyzed with unpaired $t$-test. The level of significance was $P<0.05$.

\section{Results \\ Cytokine levels}

IL-2, IL-4, IL-5, IL-10 and IFN- $\gamma$ were excluded from further statistical analysis since more than $50 \%$ of the samples were below LOD (Table 1).

The dialysate levels of the cytokines are shown in Table 2 and Fig. 2. The dialysate levels of IL-6, IL-7, IL-8, and IL13 were higher in patients than controls during the entire microdialysis. IL-6, IL-8 and IL-13 changed during microdialysis in both groups, while IL- $1 \beta$, IL-7 and GM-CSF changed only in patients. IL-6 and IL- 8 increased in response to tooth-clenching in both groups, while IL-7, IL-13 and TNF only increased in the patients. There were no significant differences in cytokine levels between patients and controls after tooth-clenching (160 min).

\section{Pain intensity and levels of fatigue}

Patients had higher baseline pain intensity and fatigue than controls. Tooth-clenching increased pain intensity in patients and evoked mild pain in controls. It also increased fatigue in both groups (Table 3).

There were no significant correlations between cytokines levels and other variables $\left(P>0.102 ; \mathrm{r}_{\mathrm{s}}<0.384\right)$.

Table 1 Dialysate samples with cytokines levels below LOD

\begin{tabular}{|c|c|c|c|c|c|c|c|c|c|c|c|c|c|}
\hline & $I L-1 \beta$ & IL-2 & IL-4 & IL-5 & IL-6 & IL-7 & IL-8 & IL-10 & IL-12 & IL-13 & TNF & $\mathrm{IFN}-\gamma$ & GM-CSF \\
\hline All & 75 (19.1) & $268(68.4)$ & 291 (74.2) & $264(67.3)$ & 68 (17.3) & $133(33.9)$ & $45(11.5)$ & 210 (53.6) & $180(45.9)$ & $140(35.7)$ & 169 (43.1) & 319 (81.4) & $178(45.4)$ \\
\hline Patients & $54(24.3)$ & $133(59.9)$ & $188(84.7)$ & $144(64.9)$ & 38 (17.1) & 82 (36.9) & $18(8.1)$ & $118(53.1)$ & $127(57.2)$ & $64(28.8)$ & $100(45.0)$ & $165(74.3)$ & $118(53.2)$ \\
\hline Controls & $21(12.4)$ & $135(79.4)$ & $103(60.6)$ & $120(70.6)$ & $30(17.6)$ & $51(30.0)$ & 27 (15.9) & $92(53.6)$ & $53(31.2)$ & $76(44.7)$ & 69 (40.6) & 154 (90.6) & $60(35.3)$ \\
\hline
\end{tabular}

Table show the dialysate samples with cytokines levels below LOD in 20 women with Temporomandibular Disorders myalgia (Patients) and 20 pain-free healthy aged-matched women (Controls). Data are presented as $\mathrm{n}=$ number of undetectable dialysate samples (\%) LOD Limits of detection, IL Interleukin, TNF Tumor necrosis factor, IFN Interferon, GM-CSF Granulocyte macrophage colony-stimulating factor. Bold figures denote cytokines with $50 \%$ of the samples below LOD (excluded from further analyses) 
Table 2 The average cytokine levels for all samples combined

\begin{tabular}{lllllllll}
\hline & $\mathrm{IL}-1 \beta$ & $\mathrm{IL}-6$ & $\mathrm{IL}-7$ & $\mathrm{IL}-8$ & $\mathrm{TNF}$ & GM-CSF & LL-12 & $\mathrm{IL}-13$ \\
\hline Dialysate levels & & & & & & & & \\
Patients & $2.3(7.0)$ & $25.8(42.3)$ & $11.4(21.5)$ & $36.2(51.4)$ & $4.4(8.8)$ & $10.1(11.2)$ & $5.5(11.8)$ & $4.1(5.9)$ \\
Controls & $1.6(2.3)$ & $11.0(12.6)$ & $6.4(3.2)$ & $10.1(14.7)$ & $3.5(1.5)$ & $14.8(23.3)$ & $7.4(3.0)$ & $9.7(13.4)$ \\
P-values & 0.208 & $\mathbf{0 . 0 1 5}$ & $\mathbf{0 . 0 4 1}$ & $\mathbf{0 . 0 0 2}$ & 0.291 & 0.199 & 0.507 & $\mathbf{0 . 0 0 2}$ \\
\hline
\end{tabular}

The average cytokine levels for all samples combined (0-220 min) in 20 women with Temporomandibular Disorders myalgia (Patients) and 20 pain-free healthy age-matched women (Controls). Data are presented as median (IQR)

IQR interquartile range (75 percentile minus 25 percentile), IL Interleukin, TNF Tumor necrosis factor, GM-CSF Granulocyte macrophage colony-stimulating factor. Bold italic figures denote significant group differences $(P<0.05)$

\section{Baseline characteristics and PPT}

Baseline characteristics of the participants and PPT before and after microdialysis are shown in Table 4. There were no differences between groups in PSS-14 or STAItrait levels, but the MVCF was lower in patients than controls. PPT over the masseter muscles were lower in patients than controls, in contrast to the reference point, but had not changed after microdialysis in any group (Table 4).

\section{Discussion}

The main findings were that the masseter levels of IL-6, IL-7, IL-8 and IL-13 were higher in TMD myalgia patients than controls and that repetitive tooth-clenching increased the levels of IL- 6 and IL- 8 in both groups, while IL-7, IL-13 and TNF increased only in patients. Also, tooth-clenching evoked higher pain intensity and fatigue in TMD myalgia patients than controls. Finally, there were no correlations between pain, fatigue and cytokine levels.

Overall, the results from this study supports the hypotheses that 1 ) the muscle levels of pro- and antiinflammatory cytokines appear to be elevated in TMD myalgia and 2) severally pro- and anti-inflammatory cytokines increased after repetitive tooth-clenching in both groups. However, there were no significant differences between TMD myalgia patients and controls. This

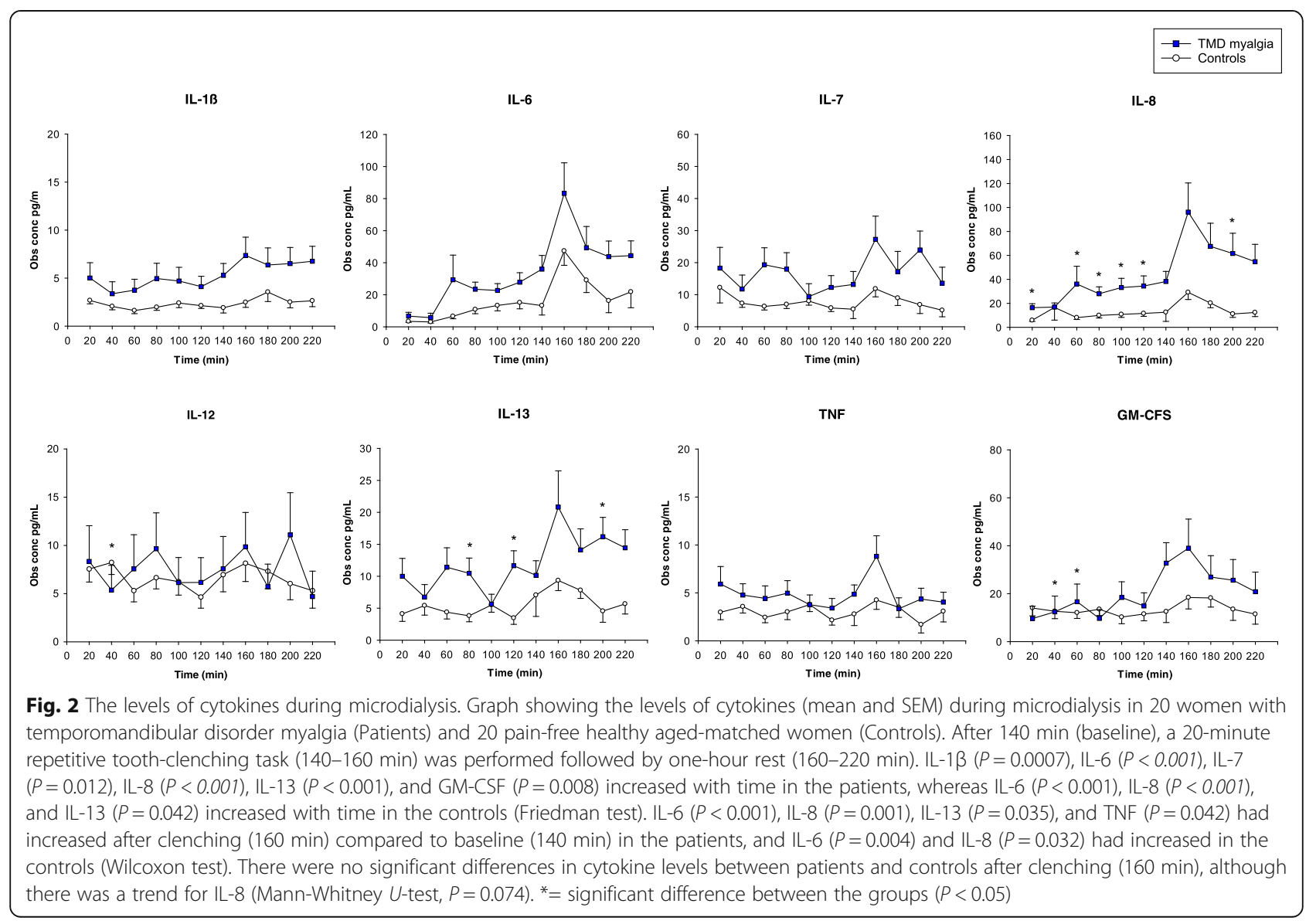


Table 3 The median (IQR) pain intensity (NRS) and fatigue (Borg RPE) in the masseter muscle

\begin{tabular}{llll}
\hline & Before & After & P-values \\
\hline Pain & $3(3)$ & $7(3)$ & $<0.001$ \\
Patients & $0(0)$ & $0(2)$ & $\mathbf{0 . 0 1 6}$ \\
Controls & $<\mathbf{0 . 0 0 1}$ & $<\mathbf{0 . 0 0 1}$ & \\
$\begin{array}{l}\text { P-values between groups } \\
\text { Fatigue }\end{array}$ & $13(4)$ & $19(1)$ & $<\mathbf{0 . 0 0 1}$ \\
Patients & $6(0)$ & $14(3)$ & $<\mathbf{0 . 0 0 1}$ \\
Controls & $<\mathbf{0 . 0 0 1}$ & $<\mathbf{0 . 0 0 1}$ & \\
P-values between groups &
\end{tabular}

Table show the median pain intensity and fatigue before and after a 20-minute tooth-clenching task in 20 women with Temporomandibular Disorders myalgia (Patients) and 20 pain-free healthy age-matched women (Controls). IQR interquartile range ( 75 percentile minus 25 percentile), NRS Numeric rating scale (0-10), Borgs's RPE Borg's ratings of perceived exertion scale (6-20). Bold italic figures denote significant differences $(P<0.05)$

lends support for a role of peripheral inflammation to drive chronic muscle pain. However, 3) no correlations with pain, fatigue or PPT levels were found, so in future studies the balance and interaction between pro- and anti-inflammatory cytokines as well as other inflammatory mediators need to be considered.

This is the first study to show elevated muscle levels of the pro-inflammatory cytokines IL- 6 and IL- 8 and the

Table 4 Baseline characteristics in 20 women with Temporomandibular Disorders myalgia and 20 healthy pain-free age-matched women

\begin{tabular}{llll}
\hline & Patients & Controls & $P$-values \\
\hline Age $(\mathrm{yr})$ & $31(10)$ & $29(11)$ & \\
MVCF $(\mathrm{kg})$ & $356(205)$ & $469(113)$ & $\mathbf{0 . 0 1 8}$ \\
STAl-trait (20-80) & $42(19)$ & $36(10)$ & 0.321 \\
PSS-14 (0-56) & $23(15)$ & $20(9)$ & 0.392 \\
PPT (kPa) & & & \\
$\quad$ Before & $160( \pm 70)$ & $239( \pm 62)$ & $\mathbf{0 . 0 0 3}$ \\
$\quad$ Experimental side & $163( \pm 46)$ & $230( \pm 30)$ & $<\mathbf{0 . 0 0 1}$ \\
$\quad$ Control side & 0.876 & 0.570 & \\
$\quad$ P-values between sides & $412( \pm 131)$ & $384( \pm 115)$ & 0.573 \\
$\quad$ Reference point & & & \\
After & $124( \pm 71)$ & $232( \pm 52)$ & $<0.001$ \\
$\quad$ Experimental side & $147( \pm 66)$ & $234( \pm 48)$ & $<\mathbf{0 . 0 0 1}$ \\
$\quad$ Control side & 0.298 & 0.919 & \\
$\quad$ P-values between sides & $454( \pm 273)$ & $425( \pm 94)$ & 0.741 \\
$\quad$ Reference point & &
\end{tabular}

The baseline characteristics as well as pressure pain thresholds (PPT) before and after microdialysis are shown

Data are presented as median (IQR) or mean $( \pm \mathrm{SD})$. IQR interquartile range (75 percentile minus 25 percentile), SD Standard deviation, MVCF Maximum voluntary contraction force, STAI State- and trait anxiety inventory, PSS Perceived stress scale. Bold italic figures denote significant differences $(P<0.05)$ anti-inflammatory cytokines IL-7 and IL-13 in painful jaw muscles of patients with TMD myalgia which supports the hypothesis that patients with TMD myalgia have higher levels of cytokines. The results are partly consistent with previous results in other localized myalgias showing increased IL-6 in the painful trapezius muscle of WAD patients [23] and IL-1 $\beta$ and TNF in myofascial trapezius trigger points [21,22], although one early study did not find any difference in trapezius levels of IL- 6 between patients with chronic trapezius myalgia and controls [37]. They are also consistent with very recent results showing that lipopolysaccharide (LPS) stimulated monocytes from patients with TMD show an enhanced production of IL- $1 \beta$, TNF and IL-6 [38]. Proinflammatory cytokines may act directly on nociceptor terminals to induce pain [39]. Increased muscle levels of other inflammatory markers, such as serotonin and glutamate have also been reported in chronic localized myalgia [15, 23, 35]. These results combined gives support for the involvement of peripheral mechanisms in localized chronic myalgia, and may implicate that patients with chronic pain constantly have their immune system switched on with higher levels of inflammatory mediators leading to peripheral sensitization, which may drive central sensitization processes and pain [40]. The increased muscle levels of IL-7 and IL-13 in the patients in this study imply that anti-inflammatory cytokines are locally produced to counteract this effect, although this may not be sufficient as LPS-stimulated monocytes from TMD-patients showed a blunted response of IL-10 [38].

In patients with TMD elevated plasma levels of both pro- (IL-1 $\beta$, IL-6, TNF) and anti-inflammatory (IL-10) cytokines were recently reported [20]. Contrary, in TMD patients with widespread pain and in fibromyalgia plasma levels of pro-inflammatory IL- 8 were higher $[18,19,41]$ and IL-10 lower than controls [18]. This suggests that the balance between pro- and antiinflammatory cytokines may differ between TMD with and without widespread pain [19] and supports a shift to a more pronounced central pro-inflammatory state in widespread myalgia compared to localized myalgia. Thus, peripheral sensitization may be of greater importance in the pathophysiology of localized myalgias than in widespread pain.

Tooth-clenching increased IL-6, IL-7, IL-8, TNF and IL-13 in TMD myalgia patients and IL- 6 and IL- 8 in controls, in line with previous studies [25, 37]. IL- 6 and IL-8 are regarded as myokines, i.e. cytokines that are produced in the muscle [24], while TNF probably is produced in other tissue [42]. Although plasma cytokines were not analyzed in this study, muscle levels of IL- 6 and IL- 8 (but not IL-1 $\beta$ or TNF) were reported higher than plasma levels in patients with fibromyalgia, supporting that they are produced in the muscle [25]. It 
is well described that plasma levels of IL-6 increase in response to exercise. Also, plasma levels of the antiinflammatory cytokines IL-4, IL-7, IL-10, and IL-13 seem to increase after exercise in healthy subjects [43] and IL-10 also in patients with knee-osteoarthritis [44]. On the contrary, in patients with fibromyalgia, plasma IL-10 showed a blunted response and IL-8 seem to decrease $[26,45]$. Thus, patients with localized pain may be able to recruit an anti-inflammatory response after exercise in contrast to patients with generalized pain.

The repetitive tooth-clenching task evoked pain in the controls and increased fatigue in both groups, with higher intensities in TMD myalgia patients in accordance to previous studies using the same methodology $[15,30]$. However, other mechanisms than release of cytokines are most probably responsible for this as there were no correlations between the release of cytokines on one hand, and pain or fatigue on the other. This indicates that there is no direct cause-effect relation between pain and cytokine levels. Indeed, the increased pain level may be an effect of the release of other biomarkers $[35,46]$ or interactions between several other mechanisms [47].

Pain on palpation, reflecting muscle allodynia, is one of the key symptoms of chronic muscle pain why it was not surprising that PPT were lower in patients than controls. Allodynia is generally regarded a sign of central sensitization [48], but peripheral sensitization may participate to the lowered PPT, as there were no differences between TMD myalgia patients and controls in PPT over the reference point. However, masseter PPT did not change in response to tooth-clenching and did not correlate with cytokine levels. This could be the reason why the influence of muscle cytokines on muscle allodynia in TMD myalgia is probably minor, if any at all.

\section{Limitations and strengths}

This is, to our knowledge, the first study investigating the interstitial jaw-muscle release of cytokines in TMD myalgia. Another strength is that a panel with 13 proand anti-inflammatory cytokines was used for the analysis in the same dialysate samples so that differences in their pattern after tooth-clenching could be analyzed.

One limitation was that some cytokines were under LOD and had to be excluded. Yet, the most common cytokines reported in previous microdialysis studies could be detected. There are several factors that can affect the dialysate levels, such as the flow-rate, the diffusion-rate through the tissue, the area and weight cut-off of the dialysis membrane, and the composition of the perfusate [49]. Perhaps by adding a colloid to the ringer-solution, the cytokines under LOD could have been detected [50]. Another limitation was that the relative recovery was not analyzed, which could have provided a true value of the extracellular concentrations. Furthermore, the patients with TMD myalgia were quite young and had a low average pain intensity at baseline, and may therefore not fully represent the general TMD myalgia population. However, the variability in pain intensity and age differs in experimental studies of TMD patients with myalgia. The data in our study is in accordance with findings of a previous study [51]. Finally, the sample was limited only to women that were psychologically healthy why it is unclear if the results can be generalized to male patients and patients with more severe psychological co-morbidities.

\section{Conclusion}

This study showed that the muscle levels of IL-6, IL-7, IL8 and IL-13 were increased in patients with TMD myalgia and increased further in response to experimental toothclenching, as did jaw-muscle pain and fatigue. This give further support that muscle inflammation may drive chronic myalgia, but that patients with TMD myalgia have a normal anti-inflammatory response to exercise. However, the lack of correlation between pain and cytokine levels, indicates that there is no direct cause-relation effect between increased pain and cytokine release and that other peripheral mediators and mechanisms, such as central sensitization are important for pain mediation.

\section{Abbreviations \\ 5-HT: 5-hydroxytryptamine; serotonin; DC/TMD: Diagnostic Criteria for temporomandibular disorders; IFN-ץ: Interferon gamma; IL: Interleukin; NRS: Numeric rating scale; PPT: Pressure pain threshold; PSS: Perceived Stress Scale; STAI-trait: State-Trait Anxiety Inventory; TMD: Temporomandibular disorders; TNF: Tumor necrosis factor; WAD: Whiplash-associated disorders}

\section{Acknowledgements \\ Jonas Goerlash is gratefully acknowledged for assisting with microdialysis in healthy controls. \\ This study was supported by grants from the Swedish Research Council (K2009-52P-20943-03-2), the Stockholm County Council (ALF project and SOF-project), the Swedish Rheumatism Association, the Swedish Dental Association, the Department of Dental Medicine at Karolinska Institutet, and the American Dental Association in Sweden (ADSS).}

\section{Authors' contributions}

SLJ contributed to conception, study design, data acquisition, analysis, and interpretation, drafted the manuscript; NC, contributed to data analysis and interpretation, drafted the manuscript; PS, and TL contributed to conception, study design, data interpretation, critically reviewed the manuscript; ME contributed to conception, study design, analysis, and interpretation, drafted the manuscript. All authors gave final approval and agree to be accountable for all aspects of the work.

\section{Competing interests}

The authors declare that they have no competing interests.

\section{Author details}

${ }^{1}$ Section for Orofacial Pain and Jaw Function, Department of Dental Medicine, Karolinska Institutet, SE 14104, Huddinge, Sweden. ${ }^{2}$ Scandinavian Center for Orofacial Neurosciences (SCON), Huddinge, Sweden. ${ }^{3}$ Section of Orofacial Pain and Jaw Function, School of Dentistry and Oral Health, Aarhus University, Vennelyst Boulevard 9, DK-8000 Aarhus C, Denmark. ${ }^{4}$ Faculty of Odontology, Malmö University, Malmö, Sweden. 
Received: 20 December 2016 Accepted: 15 February 2017 Published online: 27 February 2017

\section{References}

1. Schiffman E, Ohrbach R, Truelove E, Look J, Anderson G, Goulet JP, List T, Svensson P, Gonzalez Y, Lobbezoo F, Michelotti A, Brooks SL, Ceusters W, Drangsholt M, Ettlin D, Gaul C, Goldberg LJ, Haythornthwaite JA, Hollender $L$, Jensen R, John MT, De Laat A, de Leeuw R, Maixner W, van der Meulen M, Murray GM, Nixdorf DR, Palla S, Petersson A, Pionchon P, Smith B, Visscher CM, Zakrzewska J, Dworkin SF, International Rdc/Tmd Consortium Network lafDR, Orofacial Pain Special Interest Group IAftSoP (2014) Diagnostic Criteria for Temporomandibular Disorders (DC/TMD) for Clinical and Research Applications: recommendations of the International RDC/TMD Consortium Network* and Orofacial Pain Special Interest Groupdagger. J Oral Facial Pain Headache 28(1):6-27. doi:10.11607/jop.1151

2. Dao $T$, LeResche $L$ (2000) Gender differences in pain. J Orofac Pain 14(3): 169-184, discussion 184-195

3. Lund JP, Lavigne GJ, Dubner R, Sessle BJ (2001) Orofacial pain - from basic science to clinical management, vol first. vol book, edited. Quintessence Publishing Co, Inc, Illinois

4. Gil-Martinez A, Grande-Alonso M, Lopez-de-Uralde-Villanueva I, Lopez-Lopez A, Fernandez-Carnero J, La Touche R (2016) Chronic temporomandibular disorders: disability, pain intensity and fear of movement. J Headache Pain 17(1):103. doi:10.1186/s10194-016-0690-1

5. Monteiro AA, Kopp S (1989) The sufficiency of blood flow in human masseter muscle during endurance of biting in the intercuspal position and on a force transducer. Proc Finn Dent Soc 85(4-5):261-272

6. Huang GJ, LeResche L, Critchlow CW, Martin MD, Drangsholt MT (2002) Risk factors for diagnostic subgroups of painful temporomandibular disorders (TMD). J Dent Res 81(4):284-288

7. Velly AM, Gornitsky M, Philippe P (2003) Contributing factors to chronic myofascial pain: a case-control study. Pain 104(3):491-499

8. Al-Khotani A, Naimi-Akbar A, Albadawi E, Ernberg M, Hedenberg-Magnusson B, Christidis N (2016) Prevalence of diagnosed temporomandibular disorders among Saudi Arabian children and adolescents. J Headache Pain 17:41. doi:10.1186/s10194-016-0642-9

9. Stauber WT (2004) Factors involved in strain-induced injury in skeletal muscles and outcomes of prolonged exposures. J Electromyogr Kinesiol 14(1):61-70. doi:10.1016/j.jelekin.2003.09.010

10. Slade GD, Ohrbach R, Greenspan JD, Fillingim RB, Bair E, Sanders AE, Dubner R, Diatchenko L, Meloto CB, Smith S, Maixner W (2016) Painful temporomandibular disorder: decade of discovery from OPPERA studies. J Dent Res 95(10):1084-1092. doi:10.1177/0022034516653743

11. Bennett GJ (2012) What is spontaneous pain and who has it? J Pain 13(10): 921-929. doi:10.1016/j.jpain.2012.05.008

12. Koutris M, Lobbezoo F, Naeije M, Wang K, Svensson P, Arendt-Nielsen L, Farina D (2009) Effects of intense chewing exercises on the masticatory sensory-motor system. J Dent Res 88(7):658-662. doi:10.1177/ 0022034509338573

13. Staahl C, Drewes AM (2004) Experimental human pain models: a review of standardised methods for preclinical testing of analgesics. Basic Clin Pharmacol Toxicol 95(3):97-111. doi:10.1111/j.1742-7843.2004.950301.x

14. Stohler CS (1999) Craniofacial pain and motor function: pathogenesis, clinical correlates, and implications. Crit Rev Oral Biol Med 10(4):504-518

15. Dawson A, Ghafouri B, Gerdle B, List T, Svensson P, Ernberg M (2015) Effects of experimental tooth clenching on pain and intramuscular release of 5-HT and glutamate in patients with myofascial TMD. Clin J Pain 31(8):740-749. doi:10.1097/AJP.0000000000000154

16. Zhang JM, An J (2007) Cytokines, inflammation, and pain. Int Anesthesiol Clin 45(2):27-37. doi:10.1097/AIA.0b013e318034194e

17. Koch A, Zacharowski K, Boehm O, Stevens M, Lipfert P, von Giesen HJ, Wolf A, Freynhagen R (2007) Nitric oxide and pro-inflammatory cytokines correlate with pain intensity in chronic pain patients. Inflamm Res 56(1):32-37. doi:10.1007/s00011-007-6088-4

18. Kadetoff D, Lampa J, Westman M, Andersson M, Kosek E (2012) Evidence of central inflammation in fibromyalgia-increased cerebrospinal fluid interleukin-8 levels. J Neuroimmunol 242(1-2):33-38. doi:10.1016/j.jneuroim. 2011.10.013

19. Slade GD, Conrad MS, Diatchenko L, Rashid NU, Zhong S, Smith S, Rhodes J, Medvedev A, Makarov S, Maixner W, Nackley AG (2011) Cytokine biomarkers and chronic pain: association of genes, transcription, and circulating proteins with temporomandibular disorders and widespread palpation tenderness. Pain 152(12):2802-2812. doi:10.1016/.jpain.2011.09.005

20. Park JW, Chung JW (2016) Inflammatory cytokines and sleep disturbance in patients with temporomandibular disorders. J Oral Facial Pain Headache 30(1):27-33. doi:10.11607/ofph.1367

21. Shah JP, Phillips TM, Danoff JV, Gerber LH (2005) An in vivo microanalytical technique for measuring the local biochemical milieu of human skeletal muscle. J Appl Physiol 99(5):1977-1984

22. Shah JP, Danoff JV, Desai MJ, Parikh S, Nakamura LY, Phillips TM, Gerber LH (2008) Biochemicals associated with pain and inflammation are elevated in sites near to and remote from active myofascial trigger points. Arch Phys Med Rehabil 89(1):16-23. doi:10.1016/j.apmr.2007.10.018

23. Gerdle B, Lemming D, Kristiansen J, Larsson B, Peolsson M, Rosendal L (2007) Biochemical alterations in the trapezius muscle of patients with chronic whiplash associated disorders (WAD) - A microdialysis study. Eur J Pain(London, England). doi:10.1016/j.ejpain.2007.03.009

24. Fischer CP (2006) Interleukin-6 in acute exercise and training: what is the biological relevance? Exerc Immunol Rev 12:6-33

25. Christidis N, Ghafouri B, Larsson A, Palstam A, Mannerkorpi K, BileviciuteLjungar I, Lofgren M, Bjersing J, Kosek E, Gerdle B, Ernberg M (2015) Comparison of the levels of Pro-inflammatory cytokines released in the vastus lateralis muscle of patients with fibromyalgia and healthy controls during contractions of the quadriceps muscle-a microdialysis study. PLoS One 10(12):e0143856. doi:10.1371/journal.pone.0143856

26. Torgrimson-Ojerio B, Ross RL, Dieckmann NF, Avery S, Bennett RM, Jones KD, Guarino AJ, Wood LJ (2014) Preliminary evidence of a blunted antiinflammatory response to exhaustive exercise in fibromyalgia. J Neuroimmunol 277(1-2):160-167. doi:10.1016/j.jneuroim.2014.10.003

27. van Selms MK, Lobbezoo F, Visscher CM, Naeije M (2008) Myofascial temporomandibular disorder pain, parafunctions and psychological stress. J Oral Rehabil 35(1):45-52. doi:10.1111/j.1365-2842.2007.01795.x

28. Larsson B, Rosendal L, Kristiansen J, Sjogaard G, Sogaard K, Ghafouri B, Abdiu A, Kjaer M, Gerdle B (2008) Responses of algesic and metabolic substances to $8 \mathrm{~h}$ of repetitive manual work in myalgic human trapezius muscle. Pain 140(3):479-490. doi:10.1016/j.pain.2008.10.001

29. Borg GA (1974) Perceived exertion. Exerc Sport Sci Rev 2:131-153

30. Louca S, Christidis N, Ghafouri B, Gerdle B, Svensson P, List T, Ernberg M (2014) Serotonin, glutamate and glycerol are released after the injection of hypertonic saline into human masseter muscles - a microdialysis study. J Headache Pain 15:89. doi:10.1186/1129-2377-15-89

31. Spielberger CD (1975) The measurement of state and trait anxiety: conceptual and methodological issues. In: Levi L (ed) Emotions-their parameters and measurement. Raven, New York, pp 713-725

32. Forsberg C, Bjorvell H (1993) Swedish population norms for the GHRI, HI and STAl-state. Qual Life Res 2(5):349-356

33. Cohen S, Kamarck T, Mermelstein R (1983) A global measure of perceived stress. J Health Soc Behav 24(4):385

34. Nordin M, Nordin S (2013) Psychometric evaluation and normative data of the Swedish version of the 10-item perceived stress scale. Scand J Psychol 54(6):502-507. doi:10.1111/sjop.12071

35. Ernberg M, Hedenberg-Magnusson B, Alstergren P, Kopp S (1999) The level of serotonin in the superficial masseter muscle in relation to local pain and allodynia. Life Sci 65(3):313-325

36. Chaturvedi AK, Kemp TJ, Pfeiffer RM, Biancotto A, Williams M, Munuo S, Purdue MP, Hsing AW, Pinto L, McCoy JP, Hildesheim A (2011) Evaluation of multiplexed cytokine and inflammation marker measurements: a methodologic study. Cancer Epidemiol Biomarkers Prev 20(9):1902-1911. doi:10.1158/1055-9965.EPI-11-0221

37. Rosendal L, Kristiansen J, Gerdle B, Søgaard K, Peolsson M, Kjaer M, Sörensen J, Larsson B (2005) Increased levels of interstitial potassium but normal levels of muscle IL-6 and LDH in patients with trapezius myalgia. Pain 119(1-3):201-209

38. King C R-DM, Wallet S, Fillingim R. (2016) Altered in vitro production of cytokines in temporomandibular disorder (TMD). Abstract PTH 232 presented at: IASP 2016 International Association for the Study of Pain (IASP): 16th World Congress of Pain; Yokohama, Japan

39. Marchand F, Perretti M, McMahon SB (2005) Role of the immune system in chronic pain. Nat Rev Neurosci 6(7):521-532. doi:10.1038/nrn1700

40. Kidd BL, Urban LA (2001) Mechanisms of inflammatory pain. Br J Anaesth 87(1):3-11

41. Kosek E, Altawil R, Kadetoff D, Finn A, Westman M, Le Maitre E, Andersson M, Jensen-Urstad M, Lampa J (2015) Evidence of different mediators of 
central inflammation in dysfunctional and inflammatory pain-interleukin-8 in fibromyalgia and interleukin-1 beta in rheumatoid arthritis. J Neuroimmunol 280:49-55. doi:10.1016/j.jneuroim.2015.02.002

42. Pedersen BK, Fischer CP (2007) Beneficial health effects of exercise-the role of IL-6 as a myokine. Trends Pharmacol Sci 28(4):152-156. doi:10.1016/j.tips. 2007.02.002

43. Andersson H, Bohn SK, Raastad T, Paulsen G, Blomhoff R, Kadi F (2010) Differences in the inflammatory plasma cytokine response following two elite female soccer games separated by a 72 -h recovery. Scand J Med Sci Sports 20(5):740-747. doi:10.1111/j.1600-0838.2009.00989.x

44. Helmark IC, Mikkelsen UR, Borglum J, Rothe A, Petersen MC, Andersen O, Langberg H, Kjaer M (2010) Exercise increases interleukin-10 levels both intraarticularly and peri-synovially in patients with knee osteoarthritis: a randomized controlled trial. Arthritis Res Ther 12(4):R126. doi:10.1186/ar3064

45. Bote ME, Garcia JJ, Hinchado MD, Ortega E (2013) Fibromyalgia: antiinflammatory and stress responses after acute moderate exercise. PLoS One 8(9):e74524. doi:10.1371/journal.pone.0074524

46. Rosendal L, Larsson B, Kristiansen J, Peolsson M, Sogaard K, Kjaer M, Sorensen J, Gerdle B (2004) Increase in muscle nociceptive substances and anaerobic metabolism in patients with trapezius myalgia: microdialysis in rest and during exercise. Pain 112(3):324-334. doi:10.1016/j.pain.2004.09.017

47. Svensson P, Kumar A (2016) Assessment of risk factors for oro-facial pain and recent developments in classification: implications for management. J Oral Rehabil. doi:10.1111/joor.12447

48. Woolf CJ (2011) Central sensitization: implications for the diagnosis and treatment of pain. Pain 152(3 Suppl):S2-15. doi:10.1016/j.pain.2010.09.030

49. Gerdle B, Ghafouri B, Ernberg M, Larsson B (2014) Chronic musculoskeletal pain: review of mechanisms and biochemical biomarkers as assessed by the microdialysis technique. J Pain Res 7:313-326. doi:10.2147/JPR.S59144

50. Helmy A, Carpenter KL, Skepper JN, Kirkpatrick PJ, Pickard JD, Hutchinson PJ (2009) Microdialysis of cytokines: methodological considerations, scanning electron microscopy, and determination of relative recovery. J Neurotrauma 26(4):549-561. doi:10.1089/neu.2008.0719

51. Shimada A, Castrillon EE, Baad-Hansen L, Ghafouri B, Gerdle B, Wahlen K, Ernberg M, Cairns BE, Svensson P (2016) Increased pain and muscle glutamate concentration after single ingestion of monosodium glutamate by myofascial temporomandibular disorders patients. Eur J Pain 20(9):1502-1512. doi:10.1002/ejp.874

\section{Submit your manuscript to a SpringerOpen ${ }^{\circ}$ journal and benefit from:}

- Convenient online submission

- Rigorous peer review

- Immediate publication on acceptance

- Open access: articles freely available online

- High visibility within the field

- Retaining the copyright to your article 